\title{
Factores involucrados en la toma de decision para la utilización de asistencia ventricular en trasplante pulmonar
}

\author{
Gutiérrez Soriano LP. ${ }^{1}$, Kling Gómez JC. ${ }^{1}$, Franco Gruntorad GA. ${ }^{1}$ \\ 1 Fundación Cardioinfantil, Bogotá, Colombia.
}

Introducción: La asistencia extracorpórea en Trasplante Pulmonar es utilizada de rutina en muchos centros alrededor del mundo, mientras que en otros como el nuestro se realiza sin asistencia; sin embargo, $30-40 \%$ de estos pacientes requerirán soporte circulatorio extracorpóreo de manera advertida o inadvertida. Multiples factores predicen el uso de asistencia intraoperatoria, pero no hay unificación de conceptos. Es importante determinar el criterio mas relevante para identificar tempranamente estos pacientes evitando el uso no advertido de asistencia, pues al compararla con la utilización advertida hay mayor compromiso metabólico y hemodinámico exacerbando la morbimortalidad.

Métodos: Estudio retrospectivo que incluyó todos los pacientes sometidos a Trasplante Pulmonar en la Fundación Cardioinfantil Bogotá-Colombia entre enero de 2014 y mayo de 2019 ( $n=29)$, sin excluir ningún paciente.

Se revisaron las bases de datos tomando de las evaluaciones pretrasplante el requerimiento de oxígeno y la distancia en el test de caminata, $\mathrm{SatO} 2, \mathrm{PaO} 2$ y $\mathrm{PaCO} 2$ en gasimetria arterial y función biventricular en ecocardiograma y cateterismo. Al inicio de la cirugía se registró la función biventricular por ecocardiografia transesofágica, presión de arteria pulmonar (Swan Ganz), valores de gasimetria arterial con sus variaciones transoperatorias, comparando el grupo que requirió asistencia ventricular y los que no; estableciendo la relevancia de cada criterio al momento de tomar la decisión de utilizar asistencia. Al final de la cirugía se evaluó el impacto metabólico del uso de asistencia ventricular no advertida, con valores de lactato.

Para el análisis estadístico se utilizo el test de Fischer y U de Mann Whitney, utilizando $\mathrm{p}<0,05$.

Resultados: Se estudiaron pacientes conedades entre 19 y 67 años (58,62\% hombres y 41,38\% mujeres), 17,24\% de los trasplantes fueron unipulmonares y $82,76 \%$ bipulmonares, requiriendo asistencia en el $20,69 \%$ de los casos. La fibrosis pulmonar fue la indicación más frecuente. No hubo relación entre los valores preoperatorios y el requerimiento de asistencia. El 20,6\% de los pacientes presentaron cambios ecocardiográficos en la función ventricular derecha al momento de la inducción con respecto al ecocardiograma prequirurgico. Todos los pacientes con disfunción ventricular derecha moderada o severa al inicio de la cirugía requirieron asistencia ventricular intraoperatoria (Chi Square: 18,0208 df:3) y tuvieron compromiso metabólico con lactatos superiores a 5,2 al finalizar la cirugía.

Conclusiones: El mayor determinante del requerimiento de asistencia ventricular intraoperatoria en trasplante pulmonar es el compromiso moderado o severo de la función ventricular derecha evaluada mediante ecocardiografía transesofagica después de la inducción anestésica, previniendo así el uso de asistencia ventricular no advertida.

https://doi.org/10.25237/congresoclasa2019.08 